\section{Experimental research on the}

\section{mechanism of chemical energy conversion to light energy under thermal induction}

\author{
Jiping Liu ${ }^{1,2 *}$, Zhuqing Fang ${ }^{2}$, Yinjie Wang ${ }^{1}$ and Jia Han $^{1}$
}

${ }^{1}$ School of Materials Science and Engineering, Beijing Institute of Technology, Beijing 100081, China

${ }^{2}$ School of Mechatronical Engineering, Beijing Institute of Technology, Beijing 100081, China

\section{Abstract}

Since the discovery of glare illuminators, considerable efforts have been devoted to achieving a breakthrough of high light intensity on the order of magnitude. In this paper, we prepared strong flash blinding agents for the first time by using aluminum powder, oxidant, and adhesive as the main materials, and tris-(8-hydroxyquinolinato) aluminum (Al2q3), triazoindolizine, or nano zinc oxide, etc. as electronic output brightener after mixing and granulation according to the developed formulation. It was discovered that the luminescence intensity was related to the thermal effect of the substance while the brightener only served as an auxiliary brightening effect to achieve energy non-destructive conversion. With the same formula, the luminescence intensities of glaze agents with $A D N$ and potassium perchlorate as oxidants were slightly higher than that of ammonium perchlorate oxidant; the brightening effect of nano-zinc oxide was slightly higher than those of tris-(8-hydroxyquinolinato) aluminum (Al2q3) and triazoindolizine. The luminescence intensity of the substance with a high thermal effect value was high, but the luminescence time was slightly short. Under identical conditions, the luminescence effect of nano-aluminum powder was obviously better than that of micro-aluminum powder with the highest luminescence intensity of $3.9 \times 10^{10} \sim 1.9 \times 10^{11} \mathrm{~cd}$ and the luminescence time of $39-48 \mathrm{~ms}$. The effects of shell material and structure and the effect of heat-induced mode on the luminescence intensity were also investigated. The luminescence intensity of the glare agent with a high shell strength was high, but the luminescence time was slightly short. Moreover, the energy level of the brightener is excited under the induction of high temperatures, which leads to a blue shift to promote the chemical reaction of the material in a favorable direction. Finally, the optical radiation of the thermally induced high-temperature combustion system was analyzed from the aspects of thermal effect, combustion temperature, and chemiluminescence effect. A way to improve the optical radiation intensity of a high-temperature combustion system was proposed.

\section{More Information}

*Address for Correspondence: Jiping Liu, School of Materials Science and Engineering, Beijing Institute of Technology, Beijing 100081, China, Email: liujp@bit.edu.cn

Submitted: February 17, 2021

Approved: March 1, 2021

Published: March 2, 2021

How to cite this article: Liu J, Fang Z, Wang Y, Han J. Experimental Research on the mechanism of chemical energy conversion to light energy under thermal induction. Int J Phys Res Appl. 2021; 4: 001-005.

DOI: 10.29328/journal.ijpra.1001033

Copyright: @ $2021 \mathrm{Liu} \mathrm{J}$, et al. This is an open access article distributed under the Creative Commons Attribution License, which permits unrestricted use, distribution, and reproduction in any medium, provided the original work is properly cited.

Keywords: Thermal effect chemical energy; Light energy; Optical radiation; Chemiluminescence

Check for updates

OPEN ACCESS

\section{Introduction}

The production of light includes natural light, thermal radiation light, electroluminescence, photoluminescence, chemiluminescence and bioluminescence [1-4]. The luminescent system is a reaction system that includes the conversion of chemical energy and light energy, that is, the chemical substance itself contains high chemical energy, which is mostly converted into light energy through the combustion reaction, and then released in the form of strong radiant light $[5,6]$. Later, people discovered that the illuminating light was controllable in electroluminescence, and the illuminance could be enhanced by chemical substances $[7,8]$. After exploring the materials in the d track [5], we obtained high-quality organic electroluminescent diodes (OLED), which provided humans with high-quality, durable, and low-cost lighting [9-11].

Inert gas can produce plasma effect under the conditions of explosive shock compression [12], high temperature and high-pressure plasma effect will produce tens of thousands of degrees or even hundreds of thousands of degrees of high temperature, extremely strong optical radiation (such as explosive electromagnetic wave plasma laser ammunition) [13]. Explosion excitation can emit plasma light and ultraviolet light at the same time [14], while the working fluid of the condensed phase high-temperature combustion system burns under normal pressure and emits infrared light [15].

In this paper, high-activity nano-aluminum powder, 
oxidizing agent, and adhesive are used as raw materials to cooperate with brighteners, using thermal radiation to induce electron excitation luminescence, and the wavelength is $420 \mathrm{~nm} \sim 760 \mathrm{~nm}$, the highest luminous intensity is $3.9 \times 10^{10} \sim 1.9 \times 10^{11} \mathrm{~cd}$, and the luminescence time $39 \sim 48$ $\mathrm{ms}$, the luminous working medium realizes the electronic output control and brightness enhancement. The discovery in this study is of great significance for improving the lighting effect of existing illuminating flare and for developing nonlethal new ammunition for special purposes.

\section{Experimental equipment, devices and processes}

The experimental materials include nano-scale aluminum, potassium perchlorate, brightener, glue, etc. The preparation process is as follows. Spherical aluminum powder $40-80 \mathrm{~nm}$ and oxidant were mixed according to the formula ratio. After a $2 \% \sim 5 \%$ brightener was added to the resulting mixture, the mixture was mixed homogeneously according to the dry mixing method. The homogeneous mixture was transferred to a container, in which the mixture was mixed with $0.6 \%-0.85 \%$ of glue (as a percentage by weight of solids). Through the processes of kneading, granulating, and vacuum drying, strong light blinding agent dry granular was obtained with total volatiles less than $1.0 \%$. The dry granular was filled in a constrainable shell consisting of a deferred ignition tube and a high-energy pyrotechnic charge, which was placed in a mace-shaped fire-transfer mechanism made of celluloid. When the ignition tube is applied, the flame is sprayed to heat and ignite the high-energy pyrotechnics. The flame generated by the high-energy pyrotechnics rapidly radiates heat to the strong light-blinding agent and propagates to the depth through the mace-shaped fire transfer system. As a result, explosive luminescence occurs under thermal and pressure excitation conditions. The luminescence intensities of the $100 \mathrm{~g}$ and $200 \mathrm{~g}$ glare blinding agents were examined experimentally, and the light intensities at $10 \mathrm{~m}$ to $100 \mathrm{~m}$ were recorded by a photometer and a Kistler transient recorder. The test device for exciting the strong light by thermal radiation is shown in figure 1.

\section{Results}

Table 1 records the process of thermal radiation excitation and the luminous intensity. The light intensity test results show that the luminous intensity of nano-aluminum powder, $\mathrm{KClO}_{4}, \mathrm{ADN}$, etc. are used as oxidants, and triazoindolizine and nano-zinc oxide are used as brighteners to form a strong light system. The luminous intensity reaches $10^{8} \mathrm{~cd} \sim 10^{11} \mathrm{~cd}$, followed by other materials. As the luminous intensity increases, the luminous time decreases.

\section{Discussion}

Combining the experimental results, we will discuss the optical radiation of the high-temperature combustion system from the perspectives of thermal effect, combustion

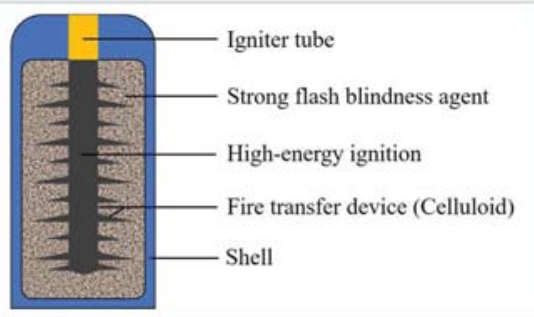

(a)

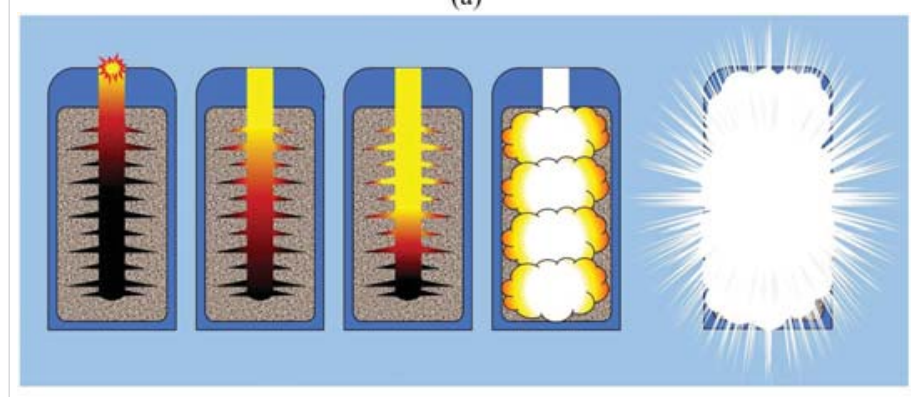

(b)

Figure 1: Test device for exciting strong light by thermal radiation and excitation glare process. (a) Test device for excitation of intense light by thermal radiation. (b) The process of thermal radiation excitation for intense light.

Table 1: The variation of luminous intensity with different material compositions.

\begin{tabular}{|c|c|c|c|}
\hline Composition & $\begin{array}{l}\text { luminous } \\
\text { intensity I (cd) }\end{array}$ & $\begin{array}{l}\text { luminous } \\
\text { time (ms) }\end{array}$ & $\begin{array}{c}\text { Wavelength } \\
\lambda(\mathrm{nm})\end{array}$ \\
\hline $\begin{array}{l}\text { 40nm spherical aluminum powder } \\
+\mathrm{KClO}_{4}+\text { triazoindolizine }\end{array}$ & $7.7 \times 10^{10}$ & 69 & 430 \\
\hline $\begin{array}{c}2 \mu \mathrm{m} \text { spherical aluminum powder }+ \\
\mathrm{KClO}_{4}+\text { triazoindolizine }\end{array}$ & $2.6 \times 10^{9}$ & 44 & 570 \\
\hline $\begin{array}{l}200 \text { mesh flake aluminum powder+ } \\
\mathrm{KClO}_{4}+\text { triazoindolizine }\end{array}$ & $3.2 \times 10^{8}$ & 41 & 710 \\
\hline $\begin{array}{l}\text { Magnesium powder }+\mathrm{KClO}_{4}+ \\
\text { triazoindolizine }\end{array}$ & $7.3 \times 10^{7}$ & 56 & 760 \\
\hline $\begin{array}{l}\text { Aluminum/magnesium alloy powder+ } \\
\qquad \mathrm{KClO}_{4}+\text { triazoindolizine }\end{array}$ & $4.3 \times 10^{8}$ & 44 & 680 \\
\hline
\end{tabular}

temperature, and chemiluminescence effect, and theoretically analyze the way to improve the luminous intensity of the hightemperature thermal radiation system through the lossless conversion of energy under thermal radiation excitation conditions.

1) The relationship between material composition and luminous effect

In addition to the temperature radiation of hot solids and liquids, the optical radiation of high-temperature combustion systems also has chemiluminescence effects. Because the combustion system is a chemical reaction system, nonequilibrium radiation output can be observed. During the chemical reaction process, some atoms or molecules may be in a higher energy state and may radiate light energy before reaching thermal equilibrium (usually through collision) [16]. The system has the potential to surpass the black body radiation, and the thermal radiation is restricted by the black body radiation, and the luminous intensity has an upper limit.

The laser is a well-known strong light source. Certain chemical reaction systems can directly exhibit laser behavior [17]. The necessary and sufficient conditions are the flipped 
distribution of the particle vibration state, that is, the number of particles in the excited state increases; the concentration of luminescent particles reaches a certain threshold, low at this value, no effective luminescence can be produced [18]. The excitation of the high-temperature combustion system may also produce the above behavior, which may provide energy beyond the thermal equilibrium or black body radiation. Due to the quantum effect and surface effect of nano-aluminum powder, the luminous intensity increases up to $10^{11} \mathrm{~cd}$, and the different types of oxidants have little effect on the luminous intensity.

2) Thermal effect and luminous intensity

The light radiation of the high-temperature combustion system is related to the heat released when the reactant burns. High thermal effect value and strong light radiation. The thermal effect of the combustion system can be calculated by Guess's law.

$$
q=\frac{\left(Q_{s}-Q_{f}\right)}{w_{n}}
$$

In the formula:

$q$ - Heat effect of the system, $\mathrm{kJ} / \mathrm{g}$;

$Q_{s}-$ Total heat of formation of products, kJ;

$Q_{f}$ - Total heat of formation of reactants, kJ;

$W_{n}-$ Total weight of the system, g.

The thermal effect of a typical binary high-energy combustion system under the conditions of zero oxygen balance (oxygen difference $n=0$ ) and negative oxygen balance $(n=-20)$, as the value of oxygen difference $n$ decreases, the thermal effect of the system increases.

Combustion temperature is an important factor affecting the light output of the system. Because the optical radiation of most high-temperature combustion systems is mainly the thermal radiation of hot solids and liquids in the flame, which is governed by the absolute black body radiation law.

$$
E=\sigma T^{4}
$$

In the formula:

$E$ - Total radiation energy, $\mathrm{W} /\left(\mathrm{m}^{2}\right)$

$\sigma-$ Stefan-Boltzman constant;

$T$ - Temperature (K).

The higher the temperature, the greater the total radiant energy. Therefore, the best light radiation system for thermal radiation effect is a system with a high combustion temperature similar to a black body.

$$
T=\frac{Q-\sum\left(Q_{s}+Q_{k}\right)}{\sum C_{p}}
$$

In the formula:

$T$ - Combustion temperature, ${ }^{\circ} \mathrm{C}$;

$Q$ - Combustion heat, kJ;

$\Sigma\left(Q_{s}+Q_{k}\right)-$ The sum of melting heat and vaporization heat of combustion products, $\mathrm{kJ}$;

$\Sigma C_{p}-$ Total heat capacity of combustion products, $\mathrm{kJ} /{ }^{\circ} \mathrm{C}_{\mathrm{o}}$

Some combustion reactions lack thermodynamic data, so it is more difficult to use the above formula to calculate. However, the molar atomic heat $\mathrm{Q}_{3}(\mathrm{~kJ} / \mathrm{mol})$ of the oxides produced by the combustible agent can be used to estimate the combustion temperature, because the temperature is proportional to $Q_{3}$ in the approximate first combustion reaction. The $Q_{3}$ values of $\mathrm{Mg}, \mathrm{Al}$, and $\mathrm{Zr}$ are 305,331 , and $364 \mathrm{~kJ} / \mathrm{mol}$, respectively. In addition, the combustion temperature can also be compared based on the melting point and boiling point of the formed oxide. The higher the melting point and boiling point of the formed oxide, the higher the combustion temperature of the system.

Judging from the $Q_{3}$ value and the melting point and boiling point of the oxide, the Zr-containing system can obtain a higher combustion temperature, and the theoretically calculated value is up to $6200{ }^{\circ} \mathrm{C}$, which is higher than that of the $\mathrm{Mg}$ containing and Al-containing systems. It has been reported in the literature that the combustion temperature of the $\mathrm{Zr}-\mathrm{O}$ system has been measured to reach $4950 \mathrm{~K}$, and is generally around 4000K [19], while the combustion temperature of the $\mathrm{Mg} / \mathrm{Al}$-containing combustion system is generally around 3500K [20]. According to Wien's displacement law, the maximum radiation wavelength is inversely proportional to the temperature $\mathrm{T}$.

$T \cdot \lambda n=\mathrm{b}\left(\mathrm{b}=2.898 \times 10^{-3} \mathrm{~m} \cdot \mathrm{K}\right)$

From the equation (4), it can be calculated that the maximum radiation wavelength of the combustion system containing $\mathrm{Mg}$ and $\mathrm{Al}$ should be about $960 \mathrm{~nm}$, which is in the infrared region; While the maximum radiation wavelength of the $\mathrm{Zr}$-containing system is about $580 \sim 725 \mathrm{~nm}$, which is just in the visible light region, which is the optical efficiency. Large area, which can produce stronger light radiation. Increase the calorific value of the material and increase the luminous efficiency. The radiation wavelength is close to the UV direction from infrared light, and the luminous intensity is the highest.

Using Planck's law, the difference in radiant energy of different systems can be seen more clearly.

$$
M_{\lambda}=2 \pi c h^{2} \lambda^{-5}\left(e^{h e / K T}\right)^{-1}
$$




$$
=C_{1} \lambda^{-5}\left(e^{c 2 / \lambda T}-1\right)^{-1}
$$

In the formula:

$M_{\lambda}$ - the radiation emission of light with a wavelength of $\lambda$ at temperature $\mathrm{T}, \mathrm{W} /\left(\mathrm{m}^{2} . \mu \mathrm{m}\right)$;

$C_{1}$ - the first radiation constant;

$C_{2}$ - the second radiation constant.

Figure 2 is the use of the above formula to make the radiant energy curve with wavelength when the temperature is $4950 \mathrm{~K}, 4000 \mathrm{~K}$ and $3000 \mathrm{~K}$.

It can be clearly seen from figure 2 that the total radiant energy has a maximum value when the radiation temperature is $3400 \mathrm{~K} \sim 4950 \mathrm{~K}$, most of the wavelengths fall within the range of $420 \sim 760 \mathrm{~nm}$, and when the temperature is below $3400 \mathrm{~K}$, it is more concentrated in the wavelength of the infrared and visible light range.

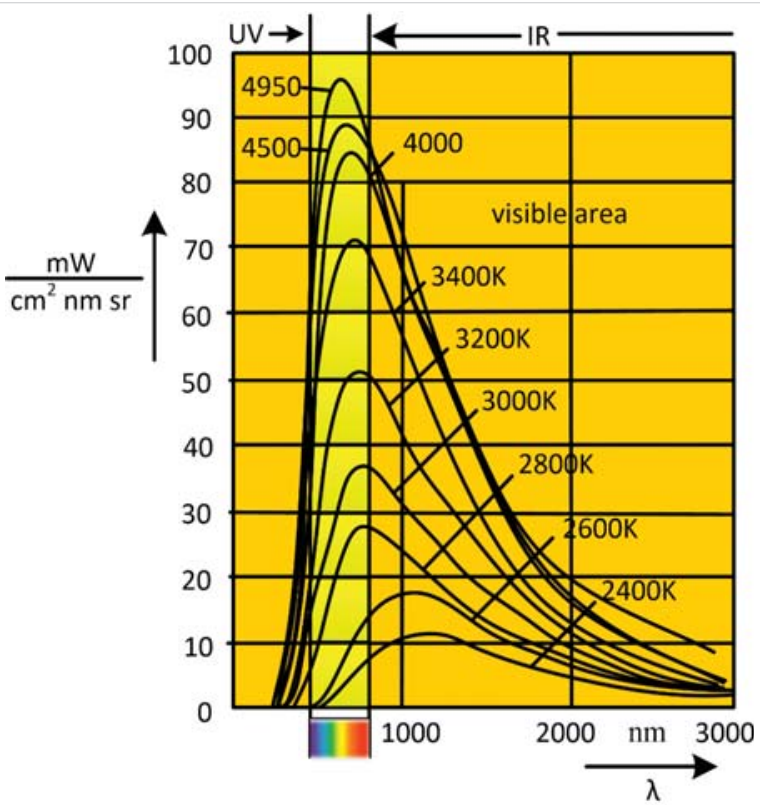

Figure 2: Curve of radiant energy versus wavelength at different temperatures.

The excitation time can be adjusted by improving the structure, which is conducive to generating a sufficiently high temperature and maintaining it for a long enough time to achieve the maximum luminous efficiency.

\section{Conclusion}

In our research, we prepared strong flash blinding agents for the first time by using aluminum powder, oxidant, and adhesive as the main materials, as well as electronic output brightener selected from tris-(8-hydroxyquinolinato) aluminum (Al2q3), triazoindolizine, or nano zinc oxide. The luminous characteristics of different media under different excitation modes are systematically studied. The factors affecting the luminous intensity are discussed separately from the aspects of thermal effect, combustion temperature, luminescent composition and photochemical effect, excitation mode and shell material.

It was discovered for the first time that the luminous intensity is related to the thermal effect of the substance. The luminous intensity with a high thermal effect value is higher, but the luminous time is slightly shorter, and the brightener only plays a role in regulating and increasing the brightness to realize energy lossless conversion. When the conditions are the same, the luminous effect of nano-aluminum powder is obviously better than that of micron-level aluminum powder, with the highest luminous intensity reaching $3.9 \times 10^{10} \sim 1.9 \times 10^{11} \mathrm{~cd}$, and the luminous time is $39 \sim 48$ $\mathrm{ms}$. The reason for the increase in luminous intensity is the quantum effect and Due to surface effects; different types of oxidants have little effect on the luminous intensity, and when the oxidant is ADN and potassium perchlorate in the same formula, the luminous intensity is slightly higher than that of ammonium perchlorate; it is found and verified that the lightenhancing effect of nano-zinc oxide is slightly higher than tris (8-hydroxyquinoline) aluminum (Al2q3) and triazoindolizine, the brightener undergoes an energy level jump induced by high temperature, resulting in the release of electronic excitation energy, and a blue shift to promote the chemical reaction of the material toward the light intensity Increase the direction of development, which is basically consistent with the results of the blue shift of the LED.

\section{References}

1. Li L, Chen Y, Zhu J. Recent Advances in Electrochemiluminescence Analysis. Anal Chem. 2017; 89: 358-371.

PubMed: https://pubmed.ncbi.nlm.nih.gov/27959507/

2. Kabe R, Adachi C. Organic long persistent luminescence. Nature. 2017; 550: 384-387.

PubMed: https://pubmed.ncbi.nlm.nih.gov/28967911/

3. Ke B, Wu W, Liu W, Liang H, Gong D, et al. Bioluminescence Probe for Detecting Hydrogen Sulfide in vivo. Anal Chem. 2016; 88: 592-595. PubMed: https://pubmed.ncbi.nlm.nih.gov/26634959/

4. Hai Z, Li J, Wu J, Xu J, Liang G. Alkaline Phosphatase-Triggered Simultaneous Hydrogelation and Chemiluminescence. J Am Chem Soc. 2017; 139: 1041-1044.

PubMed: https://pubmed.ncbi.nlm.nih.gov/28064496/

5. Moretti J, Sabatini J, Chen G. Periodate Salts as Pyrotechnic Oxidizers: Development of Barium and Perchlorate - Free Incendiary Formulations. Angew Chem Int Ed Engl. 2012; 51: 6981-6983. PubMed: https://pubmed.ncbi.nlm.nih.gov/22639415/

6. Moretti J, Sabatini J, Poret J, Gilbert R. Development of Sustainable, Epoxy-Bound $\mathrm{Mg} / \mathrm{NaNO}_{3}$ Compositions for the U.S. Army's $40 \mathrm{~mm}$ Yellow Illuminant Flares. ACS Sustain Chem Engeer. 2015; 3: 2232-2236.

7. Ferraz-Albani LA, Baldelli A, Knapp CJ, Jäger W, Vehring R, et al Enhanced evaporation of microscale droplets with an infrared laser. $J$ Heat Transfer. 2017; 139.

8. Baldelli A, Jeronimo M, Tinney M, Bartlett K. Real-time measurements of formaldehyde emissions in a gross anatomy laboratory. SN App Sci. 2020; 2: 1-13.

9. Zhang J, Duan C, Han C, Yang H, Wei Y, et al. Balanced Dual Emissions 
from Tridentate Phosphine - Coordinate Copper(I) Complexes toward Highly Efficient Yellow OLEDs. Adv Materials. 2016; 28: 5975-5979.

10. Xiang H, Li Y, Zhou L, Xie H, Li C, et al. Outcoupling-Enhanced Flexible Organic Light-Emitting Diodes on Ameliorated Plastic Substrate with Built-in Indium-Tin-Oxide-Free Transparent Electrode. ACS Nano. 2015; 9: 7553-7562.

PubMed: https://pubmed.ncbi.nlm.nih.gov/26143652/

11. Liu D, Li Y, Yuan J, Hong Q, Shi G, et al. Improved performance of inverted planar perovskite solar cells with F4-TCNQ doped PEDOT:PSS hole transport layers. J Materials Chem A. 2017; 5: 5701-5708.

12. Ditmire T, Tisch W, Springate E, Mason M, Hay N, et al. High-energy ions produced in explosions of superheated atomic clusters. Nature. 1997; 386: 54-56.

13. Ditmire T, Tisch J, Springate E. High energy ion explosion of atomic clusters: Transition from molecular to plasma behavior. Phy Rev Lett. 1997; 14: 2732-2735.

14. Kheirkhah P, Baldelli A, Kirchen P, Rogak S. Development and validation of a multi-angle light scattering method for fast engine soot mass and size measurements. Aero Sci Technol. 2020; 54: 1083-1101.

15. Davison N. 'Non-Lethal' Weapons, Palgrave Macmillan, London. 2009; 75-78.

16. Mao D, Wu W, Ji S, Chen C, Hu F, et al. Chemiluminescence-Guided Cancer Therapy Using a Chemiexcited Photosensitizer. Chem. 2017; 3: 991-1007.

17. Kao P, Liu C, Li T. Nonvolatile memory and opto-electrical characteristics of organic memory devices with zinc oxide nanoparticles embedded in the tris-(8-hydroxyquinolinato) aluminum light-emitting layer. Organic Electronics. 2015; 21: 203-209.

18. Kasper J, Pimentel. lodine-atom laser emission in alkyl iodide photolysis. Phy Rev Letters. 1965; 43: 1827.

19. Doyle W, Conway J, Grosse A. The combustion of zirconium in oxygen. J Inorganic Nuclear Chem. 1958; 6: 138-142.

20. Aly $\mathrm{Y}$, Dreizin $\mathrm{E}$. Ignition and combustion of $\mathrm{Al} \cdot \mathrm{Mg}$ alloy powders prepared by different techniques. Combustion and Flame. 2015; 162: 1440-1447. 